\title{
China boosts pandemic surveillance
}

China is stepping up disease surveillance, drug stockpiling and vaccine development as fear of a second wave of pandemic influenza H1N1 intensifies. Chinese vice-premier Li Keqiang described the country's expanded pandemic plan last week in Beijing at a meeting convened by China's health ministry, the World Health Organization (WHO) and the London-based medical journal The Lancet. Experts warn, however, that the lack of systematic screening of patients with severe respiratory conditions, and inadequate health care in the country's rural areas, may stifle the efforts.

As of 24 August, mainland China had confirmed 3,103 cases of H1N1 flu, 75\% of which were in five provinces. The only reported severe case, identified on 8 August, involves a 17-year-old in Guangdong province who also has Brugada syndrome, a genetic disease with an increased risk of sudden heart failure.

The epidemiology of H1N1 flu in China is similar to that elsewhere, says Yu Hongjie, deputy director of the disease control and

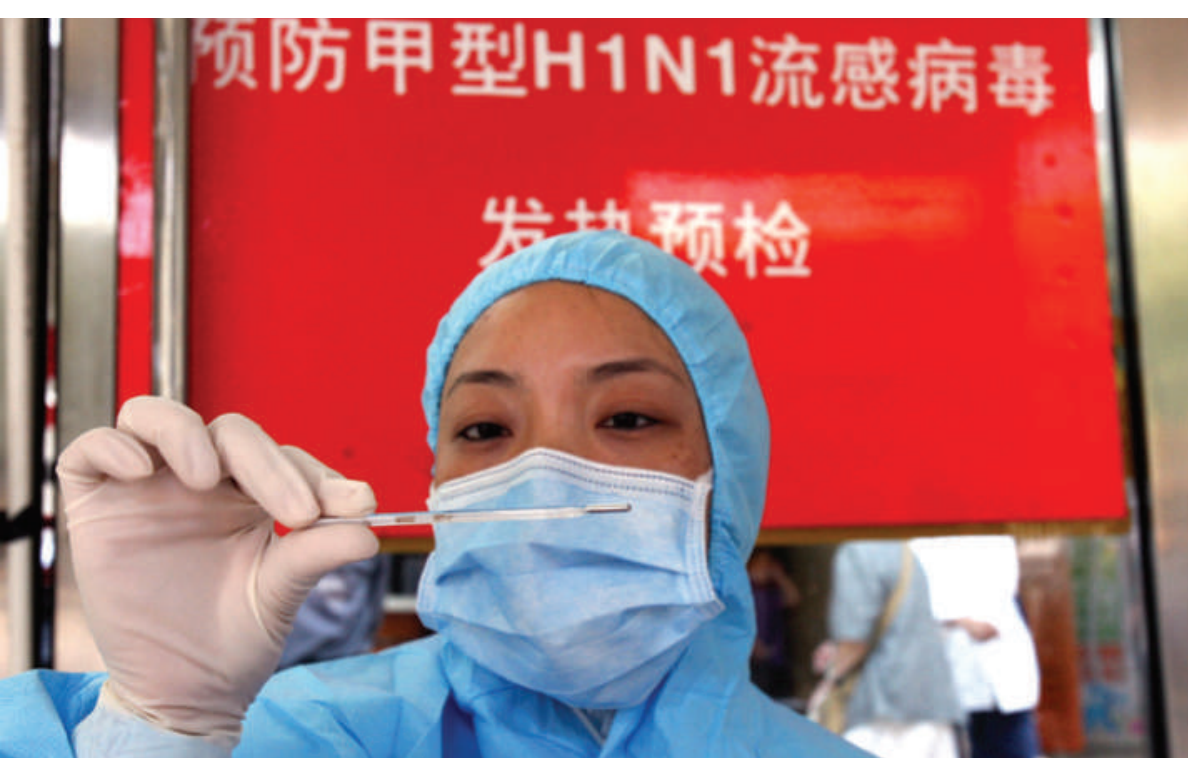

As temperatures fall, China hopes to keep the expected winter resurgence of H1N1 flu under control. emergency response office at the Beijing-based Chinese Center for Disease Control and Prevention (CDC). But of the 1.3 billion people in China, 300 million are children, elderly or pregnant, or have chronic diseases or reduced immune function - and are at high risk of developing severe or fatal disease if infected. "This is a daunting challenge and will be a test case for the country's surveillance strategies and health-care system," says Ira Longini, a biostatistician and epidemiologist at the University of Washington in Seattle.

Initially, China focused on screening those entering the country and on monitoring patients with respiratory illness who had visited areas with confirmed H1N1 cases or had been in contact with diagnosed patients. Healthy people in close contact with H1N1 patients were quarantined.

"China has been very open
"This will be a test case for the country's surveillance strategies and healthcare system." infection in the 556 'sentinel' hospitals and 411 virology laboratories across the country, says Yu. The government is closely monitoring local outbreaks and unusual disease clusters.

Some experts, such as Tomimasa Sunagawa, an epidemiologist at the National Institute of Infectious Diseases in Tokyo, are "curious" about the small number of confirmed and severe cases of H1N1 infection in mainland China compared with about 6,000 confirmed and 13 severe cases in Japan, and 8,210 confirmed and 31 severe cases in Hong Kong, as of 20 August. Chen Zhu, China's health minister, says that early diagnosis and intervention in mild H1N1 infection may have resulted in the small number of severe cases reported in mainland China.

"Although the purpose of surveillance is to follow the trajectory of the pandemic, rather than to pick up every infected individual, identifying severe from the beginning and has done an excellent job in containing the outbreak," says Keiji Fukuda, the WHO's assistant director-general for health security and environment. But the containment strategy has become inadequate as the virus continues to spread, he says. The emphasis on detection, laboratory confirmation and investigation of all cases, even mild ones, is resource-intensive, leaving little capacity to investigate severe cases and other exceptional events.

Since 8 July, China has switched to testing patients with influenza-like illness for H1N1 cases is an important component of influenza surveillance," says Robert Fontaine, a Beijingbased epidemiologist with the US Centers for Disease Control and Prevention who consults for the Chinese CDC. One approach, he says, might be to collect throat swabs from a fraction of the patients with severe respiratory conditions and test them for H1N1.

Others fear that it may be hard to monitor $\mathrm{H} 1 \mathrm{~N} 1$ infection in the rural areas. Since 2005, China has seen 36 human cases of the avian influenza H5N1 virus, with an average of eight days between disease onset and hospitalization - largely because doctors in village and county clinics are unable to make the diagnosis. In addition, doctors in underdeveloped regions may be offering inadequate or dangerous treatments for symptoms related to H1N1 infection; many clinics and hospitals in China, especially in rural areas, use dexamethasone, a potent immunosuppressant, to treat acute fever. "This could be very dangerous for patients with H1N1 infection," Fontaine says.

Wang Yu, director of the Chinese CDC, acknowledges these challenges, but says the central government is making every effort to raise pandemic awareness and the standard of diagnosis and treatment by rural doctors.

Meanwhile, China is trying to produce as much $\mathrm{H} 1 \mathrm{~N} 1$ vaccine as possible. At the Beijing meeting, Liang Xiaofeng, director of the national immunization programme at the Chinese CDC, disclosed preliminary results of vaccine trials involving more than 13,000 healthy volunteers, including the health minister. Preliminary data from nearly 4,000 people suggest that the vaccine is safe and has elicited significant immune responses after the first injection.

"The results are encouraging," says Longini. "But it's still very early days." If the findings are confirmed, says Chen, China will produce 65 million doses of vaccine by the end of the year and another 65 million by next spring.

Jane Qiu

See http://www.nature.com/swineflu for more on swine flu. 